\title{
The necessity for full ventricular coverage with noncontrast T1 mapping in hypertrophic cardiomyopathy
}

\author{
Ethan Rowin*, Martin Maron \\ From 16th Annual SCMR Scientific Sessions \\ San Francisco, CA, USA. 31 January - 3 February 2013
}

\section{Background}

Hypertrophic cardiomyopathy ( $\mathrm{HCM})$ is a genetic heart disease characterized by diverse phenotypic expression. T1 mapping is a novel CMR imaging sequence aimed at characterizing abnormal myocardial substrate. Current protocols obtain a mean $\mathrm{T} 1$ value derived from a single mid-LV short axis slice (single slice protocol) or three short-axis locations: apical, mid-LV and basal (three slice protocol). However, given the heterogeneity of disease expression within $\mathrm{HCM}$, it is unclear if $\mathrm{T} 1$ values obtained from these limited portions of the LV chamber are representative of values derived from the entire LV myocardium (global).

\section{Methods}

CMR imaging with a noncontrast $\mathrm{T} 1$ sequence using a Shortened Modified Look-Locker Inversion recovery (ShMOLLI) protocol was performed in 17 consecutive HCM patients (57 \pm 16 years old; $82 \%$ male). T1 maps of sequential $10 \mathrm{~mm}$ short axis slices from the atrioventricular ring to the apex were acquired in each patient and a mean $\mathrm{T} 1$ value was derived for each short-axis slice and then summed to generate a global LV T1 value, which were compared to mean $\mathrm{T} 1$ values derived from a single and three slice protocol.

\section{Results}

The mean global LV T1 value was $864 \pm 52 \mathrm{~ms}$ (range 731-998 ms), $850 \mathrm{~ms} \pm 59$ (range 741-942 ms) for the single segment protocol and $869 \mathrm{~ms} \pm 57$ (range 753-971 ms) for the 3 segment protocol. Throughout the entire LV chamber, there was significant variation among $\mathrm{T} 1$ values for each short-axis slice $(\mathrm{p}<0.001)$, with mean $\mathrm{T} 1$ values

significantly higher in the basal and apical levels compared to the mid-LV level $(875 \pm 56$ vs $864 \pm 65$ vs $855 \pm 52 \mathrm{~ms}$; $\mathrm{p}=0.01)$. T1 values using a single slice protocol were significantly shorter then global LV T1 values $(850 \pm 59$ vs 864 $\pm 52 ; \mathrm{p}=0.05$ ), and, while not statistically significant, $\mathrm{T} 1$ values were higher with a three slice protocol than compared to global LV values ( $869 \pm 57$ vs $864 \pm 52 ; \mathrm{p}=0.14)$.

\section{Conclusions}

In a morphologically heterogeneous disease such as $\mathrm{HCM}$, significant variations exist among T1 values across each short-axis slice of the LV myocardium. In addition, T1 values obtained using a single mid-LV short axis slice were significantly less than global T1 values. These findings support the necessity to obtain mean $\mathrm{T} 1$ values derived from the entire LV myocardium in patients with HCM.

\section{Funding}

Not applicable.

Published: 30 January 2013

doi:10.1186/1532-429X-15-S1-E1

Cite this article as: Rowin and Maron: The necessity for full ventricular coverage with noncontrast T1 mapping in hypertrophic

cardiomyopathy. Journal of Cardiovascular Magnetic Resonance 201315 (Suppl 1):E1.

Hypertrophic Cardiomyopathy Center, Tufts Medical Center, Boston, MA, USA 